Association for Information Systems AIS Electronic Library (AISeL)

Wirtschaftsinformatik Proceedings 2003

Wirtschaftsinformatik

September 2003

\title{
Erfolgsfaktoren virtueller Gemeinschaften aus Sicht von Mitgliedern und Betreibern - Eine empirische Untersuchung
}

Jan Marco Leimeister

Universität Hohenheim, leimeister@acm.org

Pascal Sidiras

Universität Hohenheim

Helmut Krcmar

Technische Universität München

Follow this and additional works at: http://aisel.aisnet.org/wi2003

\section{Recommended Citation}

Leimeister, Jan Marco; Sidiras, Pascal; and Krcmar, Helmut, "Erfolgsfaktoren virtueller Gemeinschaften aus Sicht von Mitgliedern und Betreibern - Eine empirische Untersuchung" (2003). Wirtschaftsinformatik Proceedings 2003. 86.

http://aisel.aisnet.org/wi2003/86

This material is brought to you by the Wirtschaftsinformatik at AIS Electronic Library (AISeL). It has been accepted for inclusion in Wirtschaftsinformatik Proceedings 2003 by an authorized administrator of AIS Electronic Library (AISeL). For more information, please contact elibrary@aisnet.org. 
In: Uhr, Wolfgang, Esswein, Werner \& Schoop, Eric (Hg.) 2003. Wirtschaftsinformatik 2003: Medien - Märkte - Mobilität, 2 Bde. Heidelberg: Physica-Verlag

ISBN: 3-7908-0111-9 (Band 1)

ISBN: 3-7908-0116-X (Band 2)

(c) Physica-Verlag Heidelberg 2003 


\section{Erfolgsfaktoren virtueller Gemeinschaften aus Sicht von Mitgliedern und Betreibern - Eine empirische Untersuchung}

\section{Jan Marco Leimeister, Pascal Sidiras}

Universität Hohenheim

\section{Helmut Krcmar}

Technische Universität München

Zusammenfassung: Virtuelle Gemeinschaften sind seit langem Gegenstand der Forschung. Zwar gibt es in der Literatur zahlreiche Handlungsleitfäden zu Aufund Ausbau sowie Betrieb virtueller Gemeinschaften, eine empirische Absicherung der dort postulierten Erfolgsfaktoren bleiben die meisten Arbeiten aber schuldig. Weiterhin fehlen gesicherte Erkenntnisse zu grundsätzlichen Präferenzen und Prioritätsunterschieden einzelner Anspruchsgruppen in virtuellen $\mathrm{Ge}$ meinschaften. In dieser Arbeit werden mittels einer Online-Umfrage unter Mitgliedern und Betreibern virtueller Gemeinschaften die in der Literatur vorzufindenden Erfolgsfaktoren bewertet, um darauf aufbauend mögliche Abweichungen identifizieren und analysieren zu können. Auf diese Art sollen erste empirisch gestïtzte Einblicke in Wirkungszusammenhänge und Erfolgsfaktoren bei Aufbau und Betrieb virtueller Gemeinschaften erzielt werden. Die Ergebnisse werden in Form von 10 Thesen zum Aufbau und Betrieb virtueller Gemeinschaften zusammengefasst.

Schlüsselworte: Virtual Community, virtuelle Gemeinschaft, Erfolgsfaktoren, Online-Befragung, Thesen für Aufbau und Betrieb

\section{Zielsetzung, Methodik und Aufbau der Arbeit}

Virtuelle Gemeinschaften ${ }^{1}$ sind schon seit geraumer Zeit Gegenstand der Forschung. Zwar gibt es zahlreiche Veröffentlichungen [bspw. ArHa95, ArHa96; Brun $^{+}$00; Bull02; Kim99; Pree00; Rhei93] die sich mit der Thematik befassen, aber die Hintergründe, Herangehensweisen und Fokusse sind divergent. So gibt es 
in der Literatur zwar viele Handlungsleitfäden zu Auf- und Ausbau sowie Betrieb virtueller Gemeinschaften (vgl. hierzu auch die o.g. Arbeiten); eine empirische Absicherung der dort postulierten Erfolgsfaktoren bleiben die meisten Arbeiten aber schuldig. Weiterhin fehlen gesicherte Erkenntnisse zu grundsätzlichen Präferenzen und Prioritätsunterschieden einzelner Anspruchsgruppen in virtuellen Gemeinschaften.

Ziel dieser Arbeit ist es, in der Literatur vorzufindende Erfolgsfaktoren virtueller Gemeinschaften aus Sicht von Mitgliedern und Betreibern bewerten zu lassen um darauf aufbauend mögliche Abweichungen identifizieren und analysieren $\mathrm{zu}$ können. Auf diese Art sollen erste empirisch gestützte Einblicke in Wirkungszusammenhänge bei Entwicklung, Einführung und Betrieb virtueller Gemeinschaften erzielt werden

Ausgehend von Kapitel 2, das der Klärung zentraler Begrifflichkeiten und der Offenlegung der aus Literatur und Expertenbefragungen zugrunde gelegten Erfolgfaktoren virtueller Gemeinschaften dient, wird in Kapitel 3 die Methodik der Untersuchung vorgestellt. Kapitel 4 beschreibt die Durchfuihrung der Datenerhebung und Kapitel 5 setzt sich mit der Analyse der empirischen Daten auseinander. Der Beitrag endet mit einem Fazit und gibt einen Ausblick au weitergehende Forschungsbedarfe. Im Anhang finden sich die nach Adressatengruppen unterteilten, bewerteten und gerankten Erfolgsfaktoren.

\section{Begriffliche Grundlagen und Bezugsrahmen}

\subsection{Darstellung des Begriffs „Erfolgsfaktor“}

Grundlage der Erfolgsfaktorenforschung ist die Suche nach Methoden und Modellen zur Erklärung von (Unternehmens-) Erfolg und zu dessen Maximierung Es wird nach einer möglichst genauen Anleitung für die optimale Bereitstellung und Verwendung von Ressourcen gesucht, die jedoch auf Grund der Zahl der Einflussgrößen und deren Zusammenhängen nur unzureichend erfasst werden können. Ziel ist die Aufstellung von Orientierungsgrundsätzen, die auf eine bestimmte Strategie mit Erfolgsaussicht hinmünden und auf grundlegenden Einflussgrößen durch den Betreiber basieren [Trom90, S.1]. Sie erheben nicht den Anspruch auf vollständige Erklärung der Zusammenhänge, sondern versuchen Denkansätze für die Neukonzeption einer möglicherweise schon ineffizienten Vorgehensweise zu formulieren. Diesem Erfolgsfaktorenbegriff folgend werden zunächst in der Literatur vorzufindende Erfolgsfaktoren für virtuelle Gemeinschaften zusammengetragen und anschliessend hinsichtlich ihrer Wichtigkeit von Mitgliedern und Betreibern bewertet.

\subsection{Darstellung des Begriffs „Virtuelle Gemeinschaft"}

In der Literatur ist keine einheitliche Definition des Begriffs „Virtuelle Gemeinschaft" zu finden [ScSc01, S.519]. Dies hat mehrere Gründe. Zum einen handelt es sich um ein Themenfeld, das mehrere Dimensionen enthält und somit aus verschiedenen Perspektiven mit unterschiedlichen Schwerpunkten definiert wird. Zum anderen gibt es immer wieder das Phänomen, dass Themen plötzlich ins Rampenlicht gestellt werden und sich „Buzzwords“ bilden, die nur schwer von Vorhandenem abzugrenzen sind [Pree00, S.9].

Tabelle 1 (in Anlehnung an [Mark02, S.31ff]) stellt exemplarisch vorhandene Definitionen und Perspektiven in einer Übersicht dar:

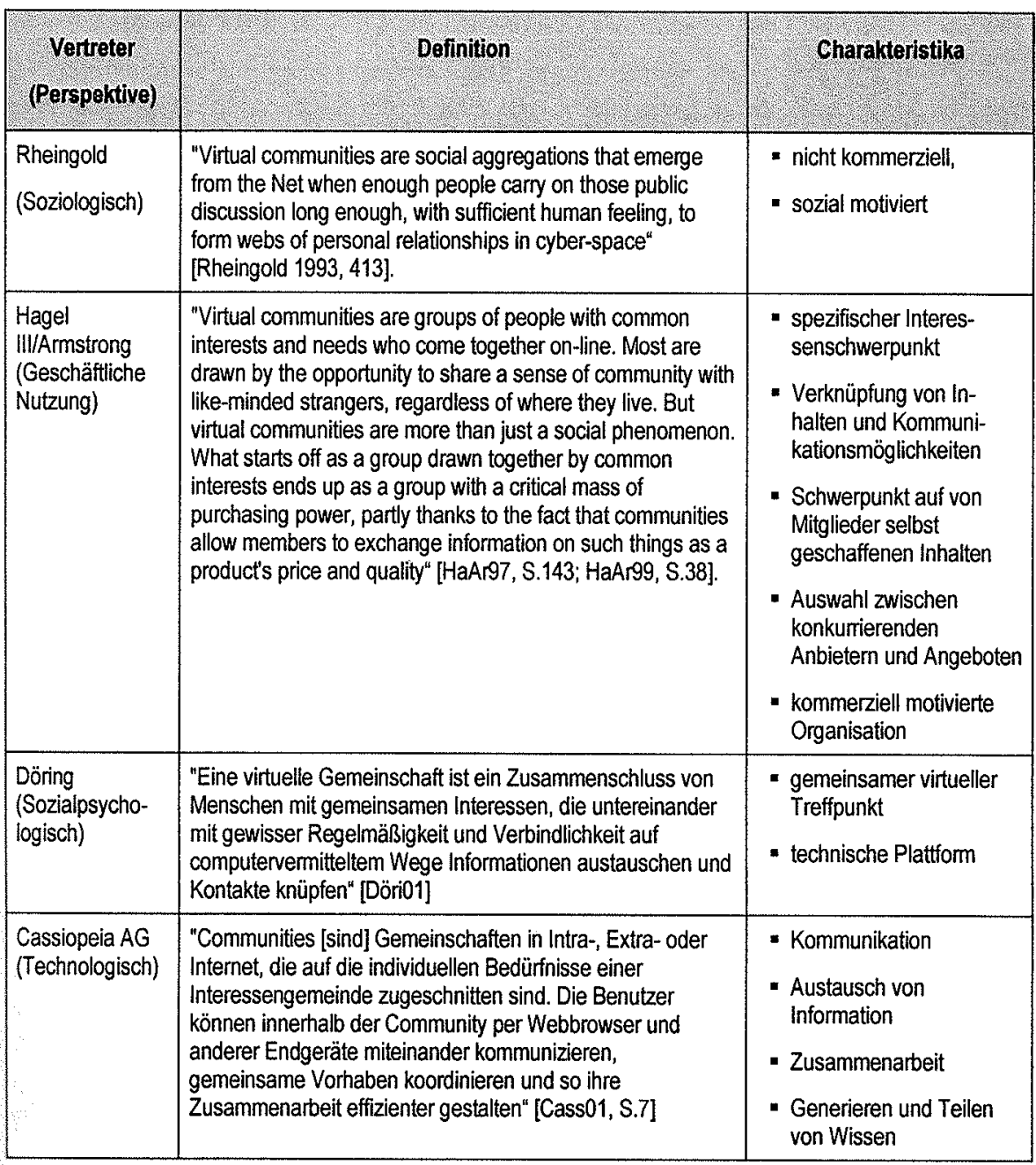




\begin{tabular}{|l|l|l|}
\hline Preece & "An online community consists of: & = interagierende Menschen \\
(Multi- & - People, who interact socially as they strive to satisfy their & " Grundregeln für diese \\
disziplinär) & own needs or perform special roles, such as leading or & Interaktion \\
& moderating. & " gemeinsamer Zweck \\
& $\begin{array}{l}\text { - A shared purpose, such as an interest, need, information } \\
\text { exchange, or service that provides a reason for the }\end{array}$ & = technische Plattform \\
& $\begin{array}{l}\text { community. } \\
\text { - Policies, in the form of tacit assumptions, rituals, protocols, } \\
\text { rules, and laws that guide people's interactions. } \\
\text { = Computer systems, to support and mediate social } \\
\text { interaction and faciliate a sense of togetherness" [Pree00, } \\
\text { S.10]. }\end{array}$ & \\
\hline
\end{tabular}

Tabelle 1: Übersicht über verschiedene Definitionen und Charakteristika virtueller Gemeinschaften

Für die Zwecke dieser Arbeit wird folgende Arbeitsdefinition zu Grunde gelegt, die ein Aggregat unterschiedlicher Definitionsmerkmale ist:

Eine virtuelle Gemeinschaft ist ein Zusammenschluss von Menschen, der durch eine technische Plattform ermöglicht wird. Grundlage und verbindendes Element der Gemeinschaft kann ein gemeinsames Interesse, ein gemeinsames Problem oder eine gemeinsame Aufgabe der Mitglieder sein, die auf Basis von impliziten oder expliziten Verhaltensregeln verfolgt werden. Die Interaktion der Mitglieder wird durch eine technische Plattform vermittelt und unterstützt, die den Aufbau von Vertrauen und einem Gemeinschaftsgefühl auch ohne die unmittelbare Präsenz ermöglicht.

\subsection{Kategorisierungsdimensionen virtueller Gemeinschaften}

Wie auch bei der Definition des Begriffes gibt es bei den Kategorisierungsdimensionen eine Vielfalt von unterschiedlichen Ansätzen.

Die Autoren Hagel/Armstrong des Klassikers " „Net Gain“ nehmen schwerpunktmäßig Bezug auf kommerzielle Communities und unterscheiden nach Zielgruppe / Zielsetzung grundsätzlich „verbraucherorientierte“ von „Unternehmen - zu Unternehmen" Communities. Verbraucherorientierte Communities werden in geografische, demografische und themenspezifische virtuelle Gemeinschaften unterteilt [HaAr99, S.134].

Bullinger et al. sehen zum Beispiel „Business Communities“ als jüngste Erscheinungsform einer Entwicklung, die von nicht-kommerziellen Interessen-

2 Vgl. hierzu auch [Schu00, S.30ff] deren Ausführungen eine Weiterführung des Ansatzes von Hagel/Armstrong darstellen. gemeinschaften über kommerzielle Communities hin zu „Geschäftsgemeinschaften für professionelles Beziehungsmanagement zwischen Kunden, Mitarbeitern und B2B-Partnern" [Bull02, S.25] reicht. Um die verschiedenen Merkmale der Kategorisierungsdimensionen herauszuarbeiten, behelfen sich Bullinger et al. mit der Frage „Wer macht Wie Was?" und folgern so auf Akteure, Werkzeuge und Ziele [Bull02, S.37])

Zupancic hingegen gliedert zwischen „Kommerziellen“ und „Nichtkommerziellen" Communities, wobei auch er die Ausdifferenzierung der kommerziellen Aspekte weiterverfolgt [Zupa99].

Brunold/Merz/Wagner unterscheiden nach der Mitgliedermotivation in die Kategorien „Informationsaustausch“, „Gemeinsame Aktivitäten“ und „Kaufen und Verkaufen" sowie in eine Sammelkategorie „Community-Plattformen und andere Sonderfälle" [Brun ${ }^{+} 00$, S.33f.]

Nach Ansicht von Figallo gliedern sich Communities entlang den Kontinua „Grad der persönlichen Interaktivität, Grad der Fokussierung und der Kohäsion der Mitglieder“, die je nach Art der Community unterschiedlich stark ausgeprägt sind [Figa98, S.6].

Bleibt festzustellen, dass bei dieser Vielzahl von Dimensionen viele real anzutreffende virtuelle Gemeinschaften nicht eindeutig zugeordnet werden können. Das kann einerseits an der besonderen Spezifität einer virtuellen Gemeinschaft liegen (so ist beispielsweise eine Community von Brustkrebspatientinnen mit starkem regionalem Fokus in der Einteilung nach Hagel III/Armstrong allen drei dort angeführten Kategorien zuzuordnen. Sie ist eine geographische Community, da ein regionaler Bezug vorhanden ist, eine demographische Community, da vorwiegend Frauen angesprochen werden und eine themenspezifische Community, da vorwiegend Brustkrebsthemen behandelt werden). Andererseits kann es daran liegen, dass selbst die vorhandenen Kategorien nicht überschneidungsfrei sind [Brun ${ }^{+} 00$, S.30].

Um die Grundgesamtheit zu untersuchender virtueller Gemeinschaften möglichst groß zu halten und um eine möglichst überschneidungsfreie Kategorisierungsmöglichkeit zu erhalten wird für die Zwecke dieser Arbeit nur nach dem finanziellen Interesse der Betreiber unterschieden, womit sich zwei Arten ergeben: Kommerzielle und nicht-kommerzielle virtuelle Gemeinschaften.

\subsection{Erfolgsfaktoren virtueller Gemeinschaften}

Analysiert man die (sehr unterschiedliche) existierende Literatur zum Thema virtuelle Gemeinschaften, so lassen sich eine Vielzahl unterschiedlicher Einflussfaktoren auf deren Erfolg identifizieren. Im Hinblick auf die Bewertung der Faktoren wird im Folgenden zwischen mitglieder- und betreiberbezogenen Erfolgsfaktoren unterschieden. Aufbauend auf [Bull02], [Figa98], [Kim99], 
[Pree00] [HaAr99] und [Brun $\left.{ }^{+} 00\right]$ wurde ein Set aus insgesamt 32 Faktoren $^{3}$ erarbeitet, von denen 26 Faktoren den Mitgliedern und alle 32 Faktoren den Betreibern virtueller Gemeinschaften zur Bewertung vorgelegt wurden (die sechs rein betreiberbezogenen Erfolgsfaktoren sind in Tabelle 2 grau hinterlegt). Die Erfolgsfaktoren wurden im Rahmen einer Delphistudie unter Experten zum Thema virtuelle Gemeinschaften überprüft, erweitert und angepasst sowie auf Korrelation getestet. Tabelle 2 gibt eine Übersicht der Erfolgsfaktoren wieder, zur besseren Identifikation der Faktoren in den Grafiken wird jedem Faktor eine Identifizierungsnummer zugeordnet

\begin{tabular}{|c|c|}
\hline Erfolgsfaktor (in Reihenfolge des Fragebogens) & Ident.-Nr. \\
\hline Erreichen einer hinreichend großen Mitgliederzahl in kurzer Zeit & 1 \\
\hline Aufbau von Vertrauen zwischen den Mitgliedern & 2 \\
\hline Entwicklung der Gemeinschaft gemäß den Vorstellungen der Mitglieder & 3 \\
\hline Angebot aktueller inhalte & 4 \\
\hline Angebot hochwertiger Inhalte & 5 \\
\hline Wertschätzung von Mitgliederbeiträgen durch den Betreiber & 6 \\
\hline Unterstützung neuer Mitglieder durch erfahrene Mitglieder & 7 \\
\hline Etablierung von Verhaltensregeln (Netiquette/ Guidelines) zur Eindämmung des Konfliktpotentials & 8 \\
\hline Unterstützung der Gemeinschaft durch regelmäßige Treffen in der realen Welt & 9 \\
\hline Sensibler Umgang mit den Daten der Mitglieder & 10 \\
\hline Durchführung regelmäßiger Events & 11 \\
\hline Intuitive Benutzerführung & 12 \\
\hline Individueller Seitenaufbau der Gemeinschaft nach Präferenzen des Mitgliedes & 13 \\
\hline Schaffung und Förderung von Untergruppen in der Gemeinschaft & 14 \\
\hline Eingliederung der Mitglieder in die Venwaltung der Gemeinschaft & 15 \\
\hline Schnelle Reaktionszeit der Webseite & 16 \\
\hline Stabilität der Webseite & 17 \\
\hline Preiseffizienz von angebotenen Produkten und Dienstleistungen & 18 \\
\hline Förderung der Interaktion zwischen Mitgliedern & 19 \\
\hline Vergabe von Vergünstigungen oder Bonusprogrammen an Mitglieder & 20 \\
\hline Vorzugsbehandlung für treue Mitglieder & 21 \\
\hline Idividuelle Angebote von Produkten und Dienstleistungen & 22 \\
\hline 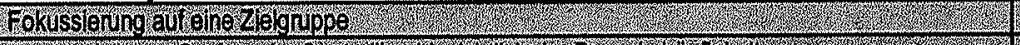 & 83 \\
\hline 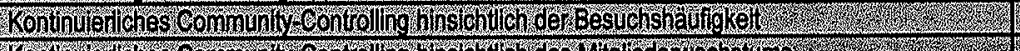 & 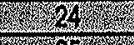 \\
\hline 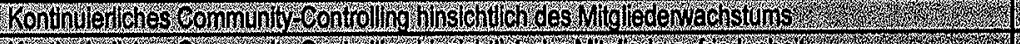 & 25 \\
\hline 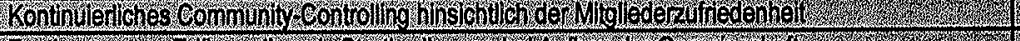 & 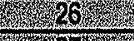 \\
\hline 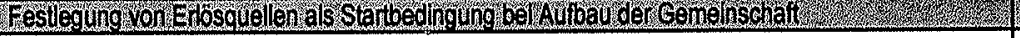 & 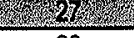 \\
\hline Ständige Erweiterung des Angebots & 28 \\
\hline 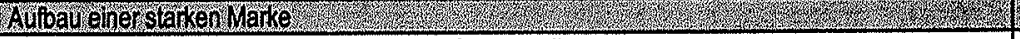 & 292 \\
\hline Die Existenz eines Offine-Kundenclubs als Startvorteil & 30 \\
\hline Erhöhung der Transparenz des Marktes für die Mitglieder & 31 \\
\hline Aufrechterhaltung der eigenen Neutralität bei der Präsentation und Auswahl der Angebote & 32 \\
\hline
\end{tabular}
vorgelegt) sind grau hinterlegt (Quelle: Eigene Darstellung).

3 Zur Ableitung der Erfolgsfaktoren sowie zur Überprüfung und Anpassung derselben mittels einer Delphistudie unter Experten siehe auch vertiefend [Sidi ${ }^{+} 03$ ]

\section{Ablauf, Aufbau und Methodik der empirischen Untersuchungen}

Aufbau und Ablauf der Untersuchung orientieren sich am Modell der Phasen de Informationsgewinnung von Nieschlag/Dichtl/Hörschgen [Nies $\left.{ }^{+} 02\right]$. Es wurde auf das vorliegende Problem übertragen und teilweise um nicht benötigte Zwischenschritte bereinigt. Abbildung 1 zeigt den Verlauf der Untersuchung.

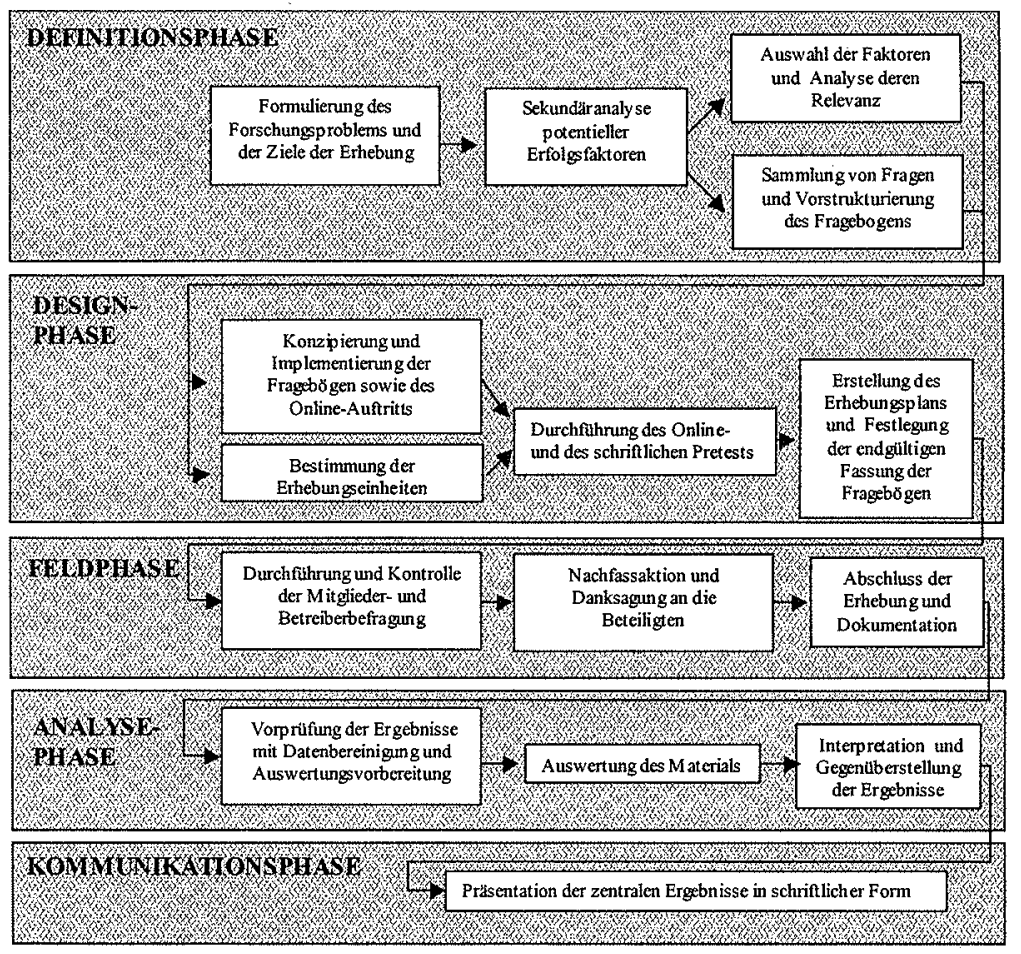

Abbildung 1: Darstellung des Untersuchungsablaufs (Quelle: Eigene Darstellung)

Für die Datenerhebung wurden ausgehend von einer Übersicht virtueller Gemeinschaften aus der Literatur [Bul102] in die noch existierenden Communities Beiträge eingestellt, die einen Aufruf zur Teilnahme an der Umfrage enthielten. Analog zu einem Schneeballverfahren wurde in diesen Communities nach Verweisen auf andere Communities gesucht und in diesen dann ebenfalls ein Beitrag mit der Bitte um Teilnahme an der Umfrage eingestellt.

Die so ermittelten 160 Communities decken sowohl nach Art als auch nach Größe der Gemeinschaften ein weites Spektrum ab. Folgend einige zusammenfassende Informationen 


\begin{tabular}{|l|l|}
\hline Zu dokumentierende Größe: & Ausprägung: \\
\hline Name des durchführenden Instituts & Lst. f. Wirtschaftsinformatik, Universität Hohenheim \\
\hline Untersuchungszeitraum & $24.07 .2002-19.08 .2002$ \\
\hline Art der Datenerhebung & Online-Umfrage \\
\hline Grundgesamtheit & K. A.4 \\
\hline Anzahl der befragten Personen & K.... \\
\hline Stichprobe & $\begin{array}{l}\text { Mitglieder: } 644 \text { (434 männl. und 210 weibl) } \\
\text { Betreiber: } 73 \text { (34 kommerz. u. 39 nicht-kommerz.) }\end{array}$ \\
\hline
\end{tabular}

Tabelle 3: Zusammenfassung der Untersuchungskennzahlen (Quelle: Eigene Darstellung)

Als Methode für diese Untersuchung wurde eine Online-Befragung gewählt, die eine besondere Form der schriftlichen Befragung darstellt. In der Literatur findet sich eine Reihe von umfangreichen Leitsätzen zur Fragebogengestaltung, denen drei immer wiederkehrende Prinzipien gemein sind: Einfachheit, Neutralität und Präzision. Zur Operationalisierung dieser Forderungen siehe auch [Büni81, S.98ff]

Die Online-Umfrage als Unterart der schriftlichen Befragung ist eine besondere Datenerhebungstechnik. Prinzipiell wird fïr die Positionierung eines Fragebogens im Internet im Vergleich zur klassischen postalischen Versendung oder der Ausgabe an ein physisch anwesendes Publikum „nur" das Präsentationsmedium gewechselt. Dieser Schritt beinhaltet aber einige Konsequenzen für den Ablauf der Untersuchung und der Gestaltung des eigentlichen Fragekatalogs, vgl. hierzu auch die Ausführungen von [Gade99, S.107ff] und [Bant ${ }^{+} 99$, S.93].

Einige Grundprobleme bleiben jedoch bei Internetumfragen bestehen: So ist die Grundgesamtheit der Internet-Nutzer im Grunde genommen undefiniert [Haup99, S.22]. Im Weiteren ist die Stichprobe in aller Regel selbstselektierend, eine aktive Stichprobenziehung zur Generierung einer repräsentativen Gruppe findet nicht statt, somit können über die „Nicht-Teilnehmer" keine Aussagen gemacht werden [Haup99, S.23].

Aufbauend auf die Sekundäranalyse zur Ermittlung von Erfolgsfaktoren virtueller Gemeinschaften (vgl. 2.4) wurde der Fragebogen in Umfang sowie Zahl der zur Bewertung stehenden Erfolgsfaktoren an die Zielgruppen angepasst, strukturiert und getestet. Dazu wurden sowohl ein klassischer Pretest mit anschließender Diskussion mit den Probanden als auch ein Online-Pretest mit Test der Funktionsfähigkeit unter denselben Gesichtspunkten durchgeführt.

Nach der Feldphase standen für die Analyse die Daten von knapp über 800 Fragebögen zur Verfügung, von denen nach der Aussortierung der unvollständig oder inkonsistent ausgefüllten Antworten 745 verwertbare Datensätze übrig

$4 \quad$ Keine genaue Zahl messbar, theoretisch alle Mitglieder und Betreiber von virtuellen Gemeinschaften weltweit

5 Nur Näherungsweise durch die "Visits" auf dem Online-Fragebogen bestimmbar, ca. 3500 Visits blieben. Durch die Aufteilung des Fragebogens in mehrere Teile (mindestens 2) und der Adressierung unterschiedlicher Teilnehmergruppen ergeben sich zahlreiche Ansatzpunkte, an denen die Gruppen der Befragten hinsichtlich ihrer Aussagen verglichen werden können. So wird auf Grund der Ergebnisse jeweils ein Ranking (s. auch [Sidi ${ }^{+}$03]) der zu bewertenden Erfolgsfaktoren aus Kapitel 3 erstellt, die Differenz zweier arithmetischer Mittel ${ }^{6}$ geprüft und mit denen der anderen Gruppen verglichen. Darüber hinaus wurden persönliche Fragen und Fragen zum Modell Community sowie der Beteiligung der Teilnehmer an diesem ausgewertet, was unabhängig von der Erfolgsfaktorenanalyse geschah. Als Grundlage der Bewertung der Erfolgsfaktoren wurde den Teilnehmern unter anderem die folgende Skala zur Verfügung gestellt (s.Abb. 2).

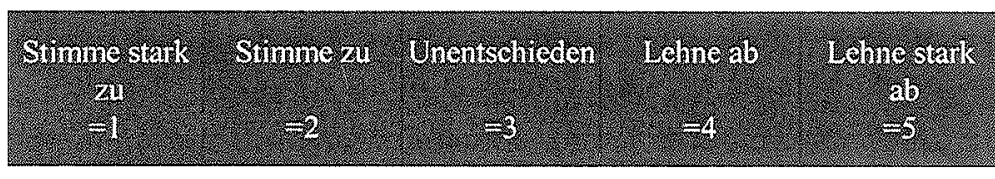

Abbildung 2: Bipolare Ordinalskala und Umdeutung in Zahlenwerte

Es wurde eine bipolare verbale Ordinalskala verwendet, die bei der Datenanalyse in die ebenfalls in Abb.2 gezeigten Zahlenwerte uminterpretiert wurde (zur Vorgehensweise siehe auch [Fahr99, S.17ff]).

Für die Untersuchung wurden aus den Datensätzen folgende 6 Gruppen gebildet, die nach der Zugehörigkeit der Beteiligten strukturiert sind: Mitglieder (alle) Mitglieder (weiblich), Mitglieder (männlich), Betreiber (alle), Betreiber (kommerziell), Betreiber (nicht-kommerziell). Diese Gruppen werden im Folgenden nach verschiedenen Gesichtspunkten miteinander verglichen.

\section{Auswertung und Vergleich der Untersuchungs- ergebnisse}

Nach einer Übersicht über die allgemeinen Aussagen der Befragten werden die Gruppen der Mitglieder und der Betreiber beschrieben und anschliessend mit- und gegeneinander verglichen.

Eine statistisch signifikante Abweichung von Mittelwerten kann erst durch ein geeignetes Testverfahren, hier der Zweistichprobentest für die Differenz zweier arithmetischer Mittel (vgl. hierzu bspw. [Voß00, S.429ff]) mit berücksichtigtem Signifikanzniveau (hier $\alpha=0,05$ ) belegt werden. 


\subsection{Allgemeine Aussagen der Befragten}

Auf die Frage nach der durchschnittlichen Verweildauer im Internet, sowohl arbeits- als auch freizeitbedingt, ergab sich bei den Mitgliedern von Communities das in Tabelle 4 gezeigt Ergebnis. Knapp die Hälfte der Online-Zeit verbringen die Personen aus der Stichprobe dabei in ihren Communities, wobei dieser Anteil bei Frauen höher liegt als bei Männern. Eine Konzentration auf nur eine Gemeinschaft kann dabei nicht beobachtet werden, im Schnitt existiert eine Mitgliedschaft bei ca. 3 Communities. Die Beitragshäufigkeit ${ }^{7}$ ist bei Männern höher als bei Frauen. Sowohl Männer als auch Frauen in der Untersuchung beziehen so gut wie nie Produkte und Dienstleistungen über ihre virtuelle Gemeinschaft. Man könnte vermuten, dass dies daran liege, dass die Mitglieder mit der Evolution „Ihrer" Community unzufrieden seien. Das kann aber verneint werden, denn sowohl Männer als auch Frauen in dieser Umfrage sind mit der Entwicklung der besuchten Community zufrieden.

\begin{tabular}{|c|c|c|}
\hline Mitgliederbefragung & Männlich & Weiblich \\
\hline Aufenthalt im Internet (Stunden/Tag) & 5,01 & 4,6 \\
\hline Aufenthalt in Community (Stunden/Tag) & 2,27 & 2,2 \\
\hline Mitglied in ... Communities & 2,98 & 2,79 \\
\hline Durchschnittliche Mitgliedschaftsdauer (Jahre) & 2,02 & 1,42 \\
\hline Beitragshäufigkeit in Community (1=mehrmals tägl., 5=nie) & 2,06 & 2,54 \\
\hline $\begin{array}{l}\text { Bezug kostenpflichtiger Produkte in/über Community (1=mehrmals } \\
\text { tägl., 5=nie) }\end{array}$ & 4,62 & 4,63 \\
\hline $\begin{array}{l}\begin{array}{l}\text { Zufriedenheit mit Entwicklung der Community ( } \uparrow=\text { sehr zufrieden, } \\
5=\text { sehr unzufrieden) }\end{array} \\
\end{array}$ & 2,27 & 2,3 \\
\hline $\begin{array}{l}\text { Anteil der Mitglieder, die andere Communitymitglieder im realen } \\
\text { Leben kennen (\%) }\end{array}$ & 52,5 & 65,1 \\
\hline
\end{tabular}

Tabelle 4: Kennzahlen und Antworten der Mitgliederbefragung (männlich: $\mathrm{n}=434$, weiblich: $\mathrm{n}=210)$;(Quelle: Eigene Darstellung).

Die an der Studie beteiligten Frauen sind durchschnittlich ein halbes Jahr kürzer Mitglied einer Community als die Männer, pflegen aber trotzdem einen regeren persönlichen (nicht mediatisierten) Kontakt. Bei einer durchschnittlichen Mitgliedsdauer von 1,42 Jahren kennen Frauen in $65,1 \%$ der Fälle mindestens ein anderes Mitglied im realen Leben, wohingegen der Anteil bei Männern bei 52,5\% liegt und die mittlere Dauer der Mitgliedschaft bei 2,02 Jahren. Obwohl keine Vergleichswerte herangezogen werden können, erscheint aber auch der geringere Wert von immerhin 52,5\% bei den Männern relativ hoch. Er bedeutet schließlich, dass im Schnitt mindestens jedes 2. Mitglied der Stichprobe, egal ob Mann oder

7 hierunter wird sowohl das Schreiben eines Beitrags im Diskussionsforum als auch das Antworten auf andere Beiträge verstanden.
Frau, ein anderes Community-Mitglied auch im realen Leben persönlich kennt. Dies könnte für eine besondere Rolle virtueller Communities beim Aufbau sozialer Beziehungen sprechen.

Die Gruppe der Betreiber (Adressaten sind hierbei die Personen, die eine Community betreiben, nicht Institutionen oder Gesellschaften) läßt sich in die Gruppe der Betreiber mit kommerziellen Interessen und in die Gruppe der Betreiber ohne kommerzielle Interessen unterteilen. Die Befragung liefert folgende Ergebnisse (vgl. auch Tabelle 5): Die Online-Zeiten der Betreiber liegen erwartungsgemäß deutlich über denen der Mitglieder. Interessant ist auch, dass kommerzielle Betreiber weniger als $50 \%$ ihrer täglichen Zeit im Netz in den von ihnen betriebenen Gemeinschaften verbringen. Dies kann nicht durch den Betrieb von mehreren Communities erklärt werden (1,82 Stück). Die Zahl der durchschnittlich betriebenen Communities liegt bei Betreibern ohne kommerzielle Interessen bei 1,26 Stück. Fragt man die Betreiber nach der Einschätzung eines möglichen Gewinn-Potentials/der Möglichkeit der Erzielung von Umsatzerlösen zeigt sich leider kein eindeutiges Ergebnis. Eine mittlere Ausprägung von 2,9 ist mit einem "Ungewiss" gleichzusetzen, eine Einschätzung die von kommerziellen und nicht-kommerziellen Betreibern geteilt wird.

\begin{tabular}{|l|c|c|}
\hline Betreiberbefragung & Kommerz. & $\begin{array}{c}\text { Nicht- } \\
\text { Kommerz. }\end{array}$ \\
\hline Aufenthalt im Internet (Stunden/Tag) & 7,28 & 6,13 \\
\hline Aufenthalt in Community (Stunden/Tag) & 3,46 & 2,68 \\
\hline Betreiber von ... Communities & 1,82 & 1,26 \\
\hline Durchschnittl.Betriebsdauer (Jahre) & 1,86 & 1,32 \\
\hline Beitragshäufigkeit in Community (1=mehrmals tägl., 5=nie) & 2,09 & 1,72 \\
\hline $\begin{array}{l}\text { Einschätzng des Gewinnpotentials der eigenen Community (1= } \\
\text { sehr gut, 5=sehr schlecht) }\end{array}$ & 2,84 & 2,97 \\
\hline $\begin{array}{l}\text { Zufriedenheit mit Entwicklung der eigenen Community (1=sehr } \\
\text { zufrieden, } \mathbf{5 = s e h r ~ u n z u f r i e d e n ) ~}\end{array}$ & 2,03 & 2,15 \\
\hline $\begin{array}{l}\text { Anteil der Betreiber, die Communitymifglieder im realen Leben } \\
\text { kennen (\%) }\end{array}$ & 73,5 & 76,3 \\
\hline
\end{tabular}

Tabelle 5: Kennzahlen und Anworten der Betreiberbefragung (kommerzielle Betreiber: $n=34$; nicht-kommerzielle Betreiber: $n=39$ ).

Bei der Frage nach der Zufriedenheit mit der Entwicklung der eigenen Community ist die durchschnittliche Zufriedenheit beider Parteien im oberen Bereich der Skala angesiedelt, bei 2,03 bzw. 2,15, was einem "Zufrieden" entspricht. Die Zufriedenheit drückt sich nach Auskunft der Befragten hierbei zu einem großen Teil in der wachsenden Zahl der Mitglieder und deren Umgang miteinander aus. Der Anteil der Betreiber, die ihre Mitglieder im realen Leben kennen ist mit mindestens $73,5 \%$ bzw. 76,3\% relativ groß. Dies mag bspw. daran liegen, dass diese Bekanntschaften durch den häufig genannten Versuch, Community-Treffen zu organisieren, entstehen. 
Die Gegenüberstellung von Einschätzungen der Gruppen der Betreiber und der Mitglieder sowie darauf aufbauend eine Analyse der Abweichungen wird in den folgenden Kapiteln 4.2 und 4.3 durchgeführt.

\subsection{Erfolgsfaktorenanalyse der Mitglieder und Vergleich der Aussagen von männlichen und weiblichen Mitgliedern}

Die Auswertung der gesamten Mitglieder hat die in Tabelle 6 dargestellte Rangordnung der Erfolgsfaktoren hervorgebracht, zusätzlich sind auch die Rangplätze der einzelnen Faktoren bei den Gruppen der Männer und Frauen separat ausgewiesen. Signifikante Abweichungen zwischen den Aussagen der Männer und Frauen sind in der letzten Spalte rot hervorgehoben.

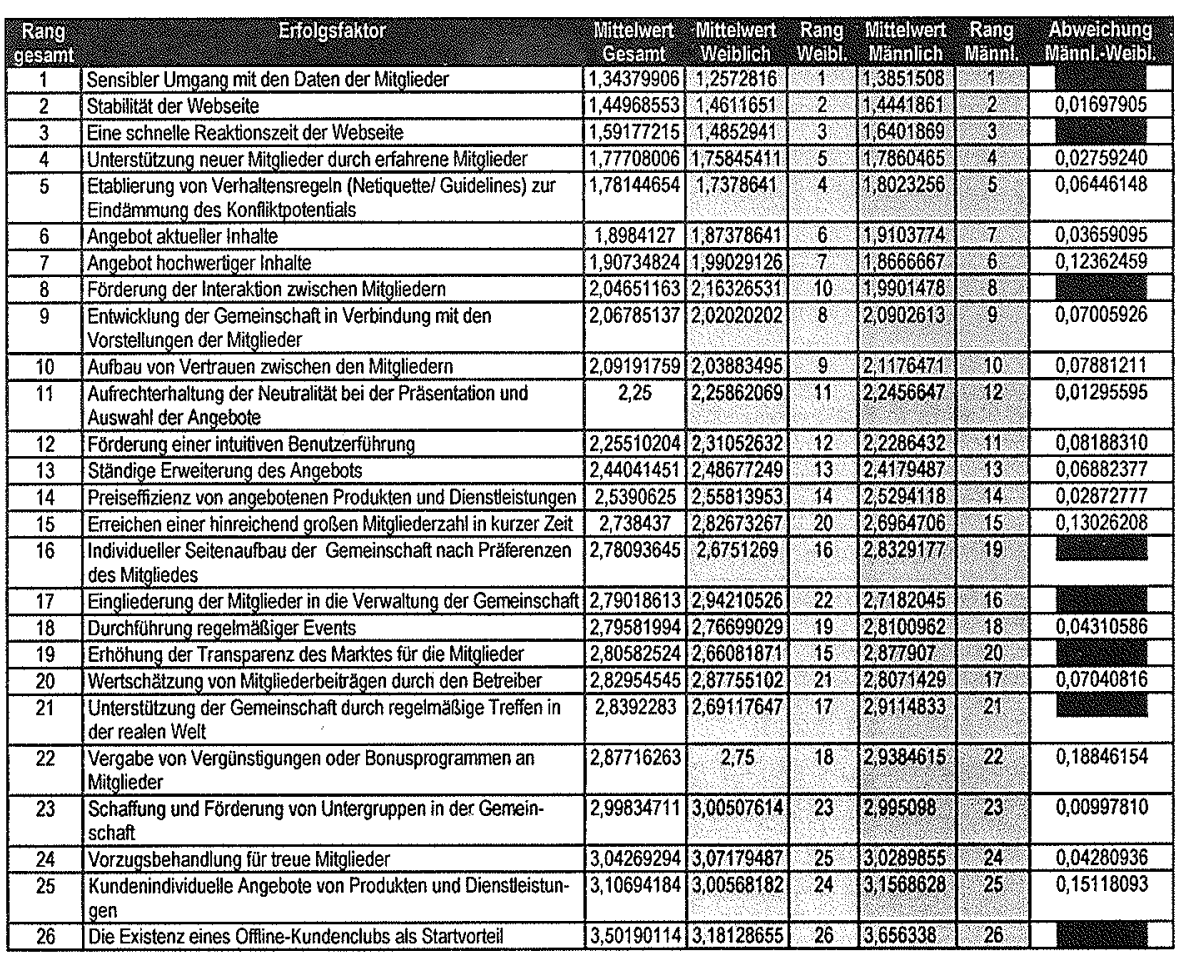

Tabelle 6: Gesamt-Rangfolge und Mittelwerte der Erfolgsfaktoren bei der Mitgliederbefragung nebst Unterteilung nach Männern und Frauen (Quelle: Eigene Darstellung)

Die Rangfolge der Faktoren zeigt als wichtigsten Erfolgsfaktor den sensiblen Umgang mit den Daten der Mitglieder, gefolgt von eher technischen Erfolgsfaktoren wie Stabilität und Reaktionszeit der Website. Neben der Rangfolge der jeweiligen Erfolgsfaktoren sind besonders die Unterschiede zwischen männlichen und weiblichen Befragten interessant und werden im Folgenden analysiert.
Signifikante (Signifikanzniveau $\alpha>0,05$ ) Abweichungen, also verschiedene Einstellungen zur Wichtigkeit der einzelnen Erfolgsfaktoren zwischen Männern und Frauen, bestehen an acht Stellen (vgl auch Tabelle 4, rot markierte Abweichungswerte).

Die Bewertung des Erfolgsfaktors "Unterstützung der Gemeinschaft durch regelmäßige Treffen in der realen Welt", erhält eine angegebene mittlere Wichtigkeit von 2,91 bei Männern bzw. von 2,69 bei Frauen. Das bedeutet, dass dieser Faktor Männern im Durchschnitt unwichtiger erscheint als Frauen. Ohne ihr Interesse bezüglich einer virtuellen Kommunikation im Allgemeinen zu berühren, drückt dies aus, dass Männern ein persönlicher Kontakt zu anderen CommunityMitgliedern relativ unbedeutender erscheint.

Diesem Zusammenhang gegenüber steht eine weitere Abweichung, die den Faktor "Förderung der Interaktion zwischen Mitgliedern" betrifft. Hier vergeben männliche User eine Wichtigkeit von 1,99, weibliche User nur 2,16. Somit ist den männlichen Usern zum einen zwar die Möglichkeit der realen Kontaktaufnahme nicht so wichtig wie den weiblichen, sie nehmen die virtuelle Interaktion aber ernster als diese. Dieses Ergebnis unterstützt die Vermutung, dass Frauen in der Community höhere Hemmschwellen bei der Kommunikation haben als Männer ${ }^{8}$, die Männer aber dafür im realen Leben weniger Wert auf die Förderung der im Netz geschaffenen Beziehungen legen (vgl. hierzu auch Tabelle 4 in Kapitel 4.1). Damit könnte sich andeuten, dass Frauen unter Umständen die Community eher zur Knüpfung neuer Beziehungen nutzen, die dann in der Realität vertieft werden können, wobei Männer mehr Wert auf den Prozess der Kontaktaufnahme, nicht aber deren Vertiefung, legen.

Die stärkste Abweichung findet sich bei der Meinung zur "Existenz eines OfflineClubs als Startvorteil“. Weibliche Nutzer sehen es als relevanter an, dass schon vor der virtuellen Gemeinschaft eine Gemeinschaft außerhalb des Internets bestanden hat, was ihre grundlegende Tendenz im vorherigen Abschnitt noch unterstreicht. Dass dieser Faktor aber in der Rangliste bei beiden betrachteten Gruppen an letzter Stelle steht, soll hier nicht verschwiegen werden. Geht es um die "Eingliederung der Mitglieder in die Verwaltung der Community", also Aufgaben die nur die virtuelle Gemeinschaft betreffen, so wendet sich das Blatt wieder: Hier wird die Wichtigkeit der Beteiligung an Verwaltungsaufgaben und ähnlichem (z.B. Moderator eines Forums) von den Männern klar in den Vordergrund gestellt, was ein Wert von 2,72 gegenüber 2,94 belegt.

Zusammenfassend wird festgestellt: Betrifft ein Erfolgsfaktor Kommunikation und Interaktion auch außerhalb des Internets, so wird er von den Frauen der Stichprobe bevorzugt, betrifft er aber das Miteinander auf der Plattform im Internet, sei es durch häufiges Beiträge schreiben, Aufgaben übernehmen oder ähnlichem, so wird er von den Männer als wichtiger erachtet. Dies lässt sich durch

\footnotetext{
8 Was sich in der deutlich geringeren Beitragshäufigkeit bestätigt, vgl. 4.1.
} 
die Antworten auf allgemeine Fragen, wie die zum Anteil der persönlichen Bekanntschaften aus der Community oder der Beitragshäufigkeit belegen (s. 4.1), aber auch durch die Analyse der Antworten auf die offenen Fragen zur Zufriedenheit und Begründung für die persönlichen Bekanntschaften.

Weitere Abweichungen kann man bei dem Faktor "Individueller Seitenaufbau der Gemeinschaft nach Präferenzen des Mitgliedes" feststellen. Die relativ geringe Abweichung, die gerade noch den Schwellenwert des Testverfahrens überschritt, kommt durch einen Mittelwert von 2,83 bei Männern und 2,68 bei Frauen zustande, was maximal zu einem 16. Rang in der weiblichen „Bestenliste“ reicht. Das tendenzielle "Unentschieden" zeigt, dass die oft unterstellten Bemühungen der Betreiber in dieser Richtung noch nicht fruchten konnten.

Bei den eher technisch orientierten Erfolgsfaktoren herrscht größtenteils Einigkeit in der Stichprobe. Eine Lücke der Mittelwerte ergibt sich jedoch bei der "Schnellen Reaktionszeit der Webseite". Die Bewertung von 1,48 bei Frauen und 1,64 bei Männern legt die Interpretation nahe, dass Männer geduldiger zu sein scheinen was die Bewegung in der Community angeht, Wartezeiten werden von ihnen eher toleriert. Dazu kommt die Einordnung dieses Kriteriums auf Rang 3 in der weiblichen Liste der wichtigsten Erfolgsfaktoren, vor dem nur noch die Stabilität der Webseite und der sensible Umgang mit den Daten der Mitglieder stehen. Komplettiert man die Betrachtung und fügt noch Aktualität und Qualität der gebotenen Inhalte an, so zeigt sich dass Frauen sehr anspruchsvoll in Bezug auf die Gestaltung und die Bedienung der Gemeinschaft sind. Die Männer in der Stichprobe unterscheiden sich in der Positionierung der Faktoren zwar nur in wenigen Punkten, die absoluten Werte liegen aber deutlich unter denen der Frauen, was deren Entschlossenheit bei der Bewertung bestätigt.

Dies zeigt auch der Unterschied beim "Sensiblen Umgang mit den Daten der Mitglieder ": Der signifikante Unterschied von 1,39 (m) zu 1,26 (w) dieses Faktors könnte auf den ersten Blick aussagen, dass Frauen die Verwendung ihrer persönlichen Daten mehr am Herzen liegen, hier wäre das aber nur eingeschränkt richtig. Der "sensible Umgang" befindet sich nämlich in beiden Stichproben klar auf Rang 1 der Liste. Es kann also einerseits zwar festgestellt werden, dass beide Parteien einen gemeinsamen eindeutigen Favoriten haben, andererseits dass die Zahlen weibliche Zielstrebigkeit und Sicherheit bezüglich der eigenen Meinung bestätigen.

\subsection{Erfolgsfaktorenanalyse der Betreiber und Vergleich der Aussagen von Betreibern kommerzieller und nicht- kommerzieller virtueller Gemeinschaften}

Dieser Vergleich überrascht durch das Ausbleiben von statistisch signifikanten Abweichungen. Trotz der somit fehlenden Voraussetzung für die Analyse der
Erfolgsfaktoren sollen die Ergebnisse hier dargestellt werden, da einige der Mittelwerte den Schwellenwert des Testverfahrens nur knapp nicht überschritten.

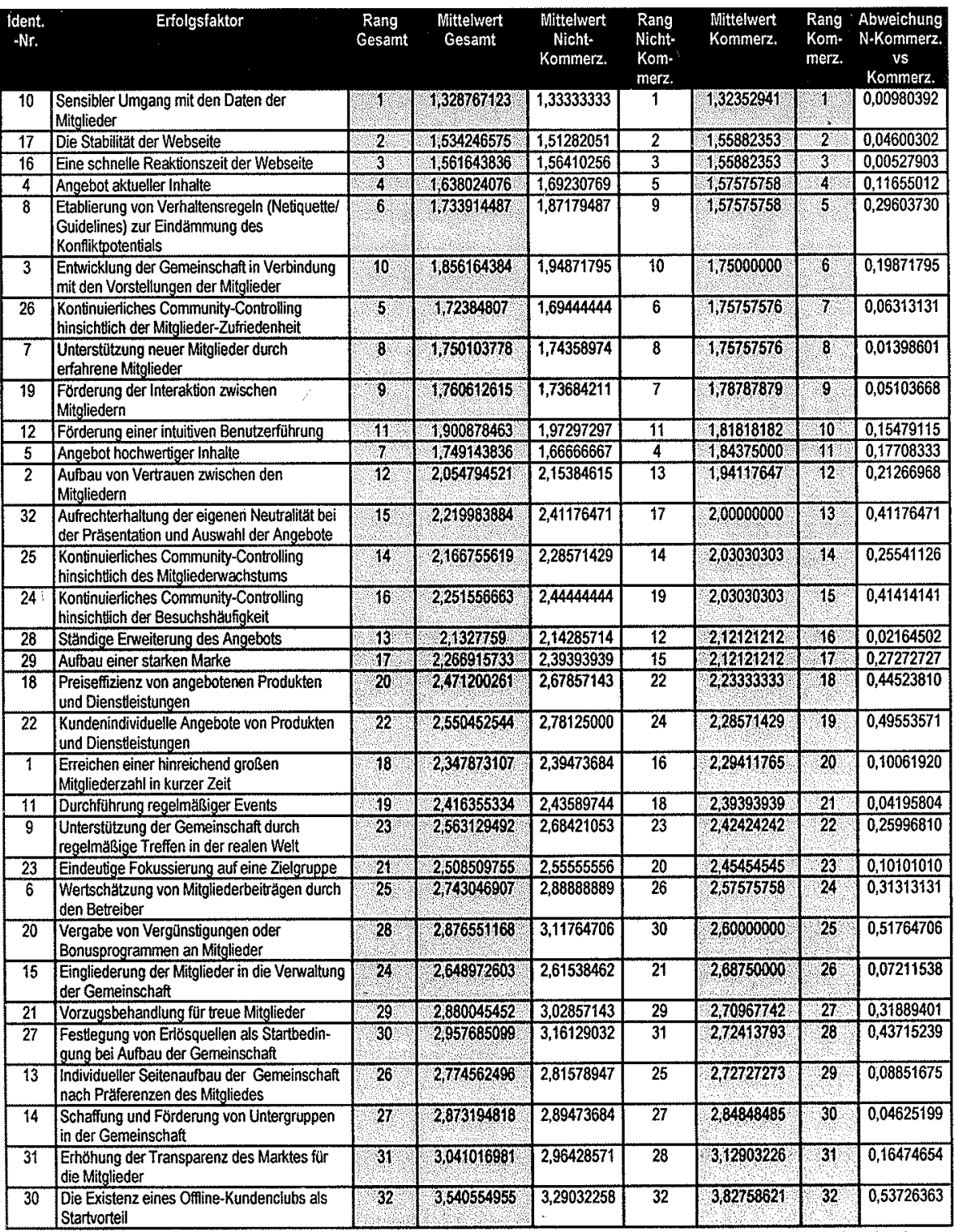

Tabelle 7: Gesamt-Rangfolge und Mittelwerte der Erfolgsfaktoren bei der Betreiberbefrag ung nebst Unterteilung nach kommerziellen und nicht-kommerziellen Betreibern, sortiert nach der Rangfolge aller Betreiber-Angaben (Quelle: Eigene Darstellung)

Jedoch fallen die Differenzen zwischen den Faktoren mit den Identifikationsnummern (Ident.-Nr.) 20 bis 25 schnell auf. Beim Testen ergeben sich an dieser 
Stelle auch die erwähnten knappen Unterschreitungen der Schwellenwerte, teilweise mit nur marginalen Unterschieden, jedoch groß genug um keine bei diesem Signifikanzniveau gültigen Aussagen zu formulieren.

So bleibt festzuhalten, dass die erwartete Konfrontation zwischen einer kommerziellen und nicht-kommerziellen Orientierung der Community zumindest auf der Ebene der Betreiber ausbleiben muss. Dieses Resultat legt den Schluss nahe, dass wenn schon der detaillierte Vergleich zwischen den Betreibern ohne nennenswerte Differenzen endete, ein ähnliches Ergebnis beim getrennten Vergleich zwischen den beiden Betreibergruppen und den Mitgliedern zu erwarten ist. Wie sich noch zeigen wird, bestehen aber besonders zwischen kommerziell orientierten Betreibern und Mitgliedern im Allgemeinen doch erhebliche Meinungsverschiedenheiten.

\subsection{Vergleich der Aussagen von Betreibern und Mitgliedern virtueller Gemeinschaften}

\subsubsection{Vergleich der Aussagen von nicht-kommerziellen Betreibern virtueller} Gemeinschaften und Mitgliedern

Wenn man voraussetzt, dass Betreiber ohne kommerzielle Ziele sich aus intrinsischer Motivation mit ,ihrer" Community auseinandersetzen, so sollten nur einige Abweichungen von den Mitgliedern bei der Relevanzbeurteilung zu Tage treten. Und tatsächlich ergeben sich nur bei folgenden zwei Faktoren statistisch signifikante Unterschiede:

- Ident-Nr.11: Durchführung regelmäßiger Events.

- Ident-Nr.19: Förderung der Interaktion zwischen Mitgliedern.

Hält man sich vor Augen, dass der am ,unwichtigsten“ beurteilte Erfolgsfaktor bei Mitgliedern mit einem Mittelwert von 3,5 bewertet wird, so erscheint die durchschnittliche Wichtigkeit von 2,8 für die "Durchführung regelmäßiger Events " sehr ernüchternd. Dies ist umso erstaunlicher, da in der Literatur Events als das Leben in der Gemeinschaft auffrischend und attraktiv auf die Mitglieder wirkend beschrieben werden [HaAr97]. Dass Betreiber dies anders sehen, zeigt die bessere Positionierung mit einer durchschnittlichen Wichtigkeit von 2,44. Trotzdem verwundert das relativ geringe Interesse der Community-Mitglieder beider Geschlechter.

Ebenfalls erstaunlich ist die Bewertung des Faktors "Förderung der Interaktion zwischen Mitgliedern". Dieser weitere, klar auf das Gemeinschaftsleben abzielende Faktor steht zwar mit einer Bewertung von 2,05 bei Mitgliedern und hohen 1,74 bei den Betreibern eindeutig besser da, die „Bevölkerung“ der
Gemeinschaft scheint aber lieber ohne unterstützenden Einfluss von Außen zu interagieren.

Das Veranstalten von gemeinsamen Events soll hier nicht als "schlecht“ eingestuft werden, wegen der Positionierung auf Rang 18 der wichtigsten Erfolgsfaktoren sollte ein Betreiber aber besser auf die höher positionierten Faktoren eingehen.

Deutlich wird durch die geringe Zahl der Abweichungen, dass eine weitgehende Einigkeit zwischen nicht-kommerziellen Betreibern und Mitgliedern virtueller Gemeinschaften besteht. Es stellt sich nun die Frage, wie sich das Bild bei klar kommerziell orientierten Betreibern wandelt, da die anfänglich festgestellten geringen Abweichungen zwischen kommerziellen und nicht-kommerziellen Betreibern anderes vermuten lassen.

\subsubsection{Vergleich der Aussagen von kommerziellen Betreibern virtueller Gemeinschaften und Mitgliedern}

Da besonders Betreiber von kommerziell orientierten Communities stark von der Zufriedenheit und der Konsumneigung sowohl ihrer Mitglieder als auch ihrer potentiellen neuen Kunden abhängig sind, ist die Vielzahl an signifikanten Abweichungen (vgl. Abb. 3) umso erstaunlicher (zur besseren Visualisierung wurde eine Polygonzug-Darstellung gewählt). Die in der vorliegenden Stichprobe vereinten Betreiber vertreten teilweise doch andere Einschätzungen in Bezug auf das was ihnen als wichtig für die Gemeinschaft erscheint als die Mitglieder. Grundsätzlich messen die Betreiber den Kriterien aber nicht weniger, sondern eher mehr Gewicht $z u$, als es die Mitglieder tun. Es stehen die folgenden Erfolgsfaktoren zur Betrachtung aus:

- Ident-Nr.1: Erreichen einer hinreichend großen Mitgliederzahl in kurzer Zeit.

- Ident-Nr.3: Entwicklung der Gemeinschaft in Verbindung mit den Vorstellungen der Mitglieder.

- Ident-Nr.4: Angebot aktueller Inhalte

- Ident-Nr.9: Unterstützung der Gemeinschaft durch regelmäßige Treffen in der realen Welt

- Ident-Nr.11: Durchführung regelmäßiger Events.

- Ident-Nr.12: Förderung einer intuitiven Benutzerführung.

- Ident-Nr.22: Kundenindividuelle Angebote von Produkten und Dienstleistungen.

Das "Erreichen einer hinreichend großen Mitgliederzahl in kurzer Zeit" liegt zwar auf einem abgeschlagenen 20. Platz in der Liste der wichtigsten Erfolgsfaktoren bei der Beurteilung durch kommerzielle Betreiber, trotzdem wird eine mittlere Wichtigkeit von 2,29 festgestellt, die einer 2,74 bei den Mitgliedern und 
somit einem 15. Rang gegenübersteht. ${ }^{9}$ Speziell für den hier betrachteten Faktor bestätigt sich die Vermutung, dass Betreibern eine große Mitgliederzahl in kurzer Zeit durch ihre Abhängigkeit vom laufenden Geschäft wichtig ist, da er mit

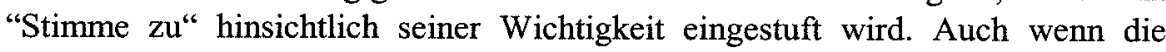
Erzielung von Erlösen in der Startphase noch nicht den erhofften Grad erreichen dürfte, so sind reger Besuch und steigende Mitgliederzahlen eine gute Voraussetzung. Die hier betrachteten Mitglieder scheinen aber eher Gemeinschaften mit geringerer Größe zu bevorzugen oder zumindest große Mitgliederzahlen nicht als Voraussetzung für ihre Teilnahme zu sehen. Dies erstaunt, wenn man sich vor Augen hält, dass die Förderung von Untergruppen von ihnen auf einen Platz 23 von 26 platziert wird. Wenn geringe Gruppengrößen vorgezogen würden, dann sollte dieser Faktor weiter vorne im Ranking zu finden sein.

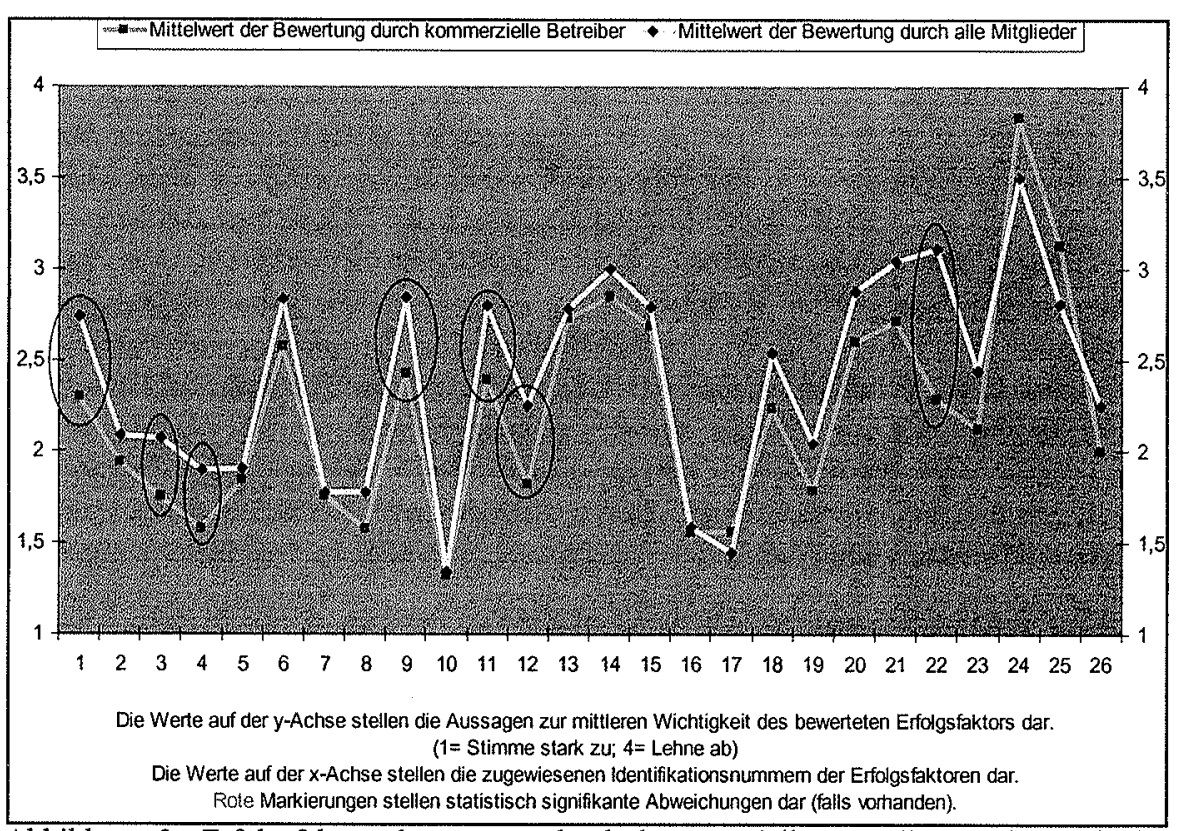

Abbildung 3: Erfolgsfaktorenbewertung durch kommerzielle Betreiber und Mitglieder (Quelle: Eigene Darstellung)

Verfolgt man nun die beiden Graphen der zu Grunde liegenden Auswertung über ihren gesamten Verlauf und genauer im Bereich der signifikant abweichenden Werte, so kann gesagt werden, dass bei jeder der hier als relevant eingestuften Abweichung die Gruppe der Betreiber eine höhere mittlere Wichtigkeit unterstellt als die eigentlichen User. Am stärksten ist dieses Phänomen bei den Faktoren "Förderung einer intuitiven Benutzerführung" und "Kundenindividuelle Angebote

9 Es gilt im Hinterkopf zu behalten, dass den Betreibern 32 Erfolgsfaktoren präsentiert wurden im Gegensatz zu den 26 bei Mitgliedern. von Produkten und Dienstleistungen “. In letzterem Fall wird der Schwellenwert der Teststatistik um mehr als das Doppelte überschritten, ein mittlerer Wert von 2,29 steht einer 3,11 auf Seiten der Mitglieder gegenüber. Ob die personalisierten Angebote störend auf die Mitglieder wirken, kann an dieser Stelle weder bestätigt noch abgelehnt werden. Dieses Ergebnis für den Erfolgsfaktor "Kundenindividuelle Angebote von Produkten und Dienstleistungen" ist für den vorliegenden Vergleich zu erwarten gewesen, auch da ein "Sensibler Umgang mit den Daten der Mitglieder" bei den Betreibern klar den 1. Platz des Rankings belegt. Die Betreiber (über-) leben aber durch den Verkauf von Produkten und Dienstleistungen unabhängig anderer eventueller Erlösquellen und messen ihnen dadurch eine hohe Bedeutung zu. Die teilnehmenden Mitglieder sind wahrscheinlich überwiegend in nicht-kommerziellen Gemeinschaften aktiv und somit äußerst kritisch gegenüber einer Kommerzialisierung.

\subsubsection{Vergleich der Aussagen von allen Betreibern und Mitgliedern}

Dieser Vergleich wirkt nach den schon abgeschlossenen Detail-Vergleichen unnötig, aber: durch den erhöhten Stichprobenumfang bei der Betrachtung der gesamten Betreiber-Gruppe, aber nur geringfügig veränderter Varianz der Aussagen, bringt das Testverfahren eine noch nicht betrachtete Abweichung hervor.

- Nr.23: Ständige Erweiterung des Angebots.

Die "Ständige Erweiterung des Angebots" wird von den Betreibern im Schnitt mit einer Wichtigkeit von 2,12, von den Mitgliedern mit 2,44 bewertet. Betreiber sehen die Erweiterung als natürliche Entwicklung des Angebots, versuchen damit zum einen sich von anderen Communities zu unterscheiden um konkurrenzfähig zu bleiben, zum anderen eventuell neue Märkte zu erschließen. Das wird von den Mitgliedern nicht als unattraktiv empfunden, bedeutet ihnen aber nicht so viel wie z.B. die "Aufrechterhaltung der Neutralität bei der Präsentation und Auswahl der Angebote"."

\section{Fazit und Ausblick}

Aus den zuvor dargestellten Ergebnissen lassen sich folgende 10 Thesen für den Aufbau und Betrieb virtueller Gemeinschaften ableiten:

These 1: Die Erstellung einer technisch performanten Plattform mit hoher Stabilität und technischer Sicherheit der Daten ist einer der wichtigsten Erfolgsfaktoren.

10 Hier kann ein Mittelwert von 2,25 festgestellt werden 
These 2: Eine Beschränkung auf Kommunikations-/Interaktionsdienste ist nur kurzfristig erfolgversprechend. Für einen nachhaltigen Erfolg einer Community gilt es zusätzlich zum User-Generated-Content inhaltlich hochwertige und aktuelle Informationen vorzuhalten.

These 3: Sensibler Umgang mit den Nutzerdaten/-profilen ist ein zentraler Erfolgsfaktor, ein Verkauf der Nutzerdaten an Dritte ist kontraproduktiv

These 4: Die Erstellung individualisierter Angebote ist in Communities nur sehr bedingt erfolgversprechend.

These 5: Das Community-Management sollte einerseits sehr schnell auf ggf. auftretende Probleme in der Community reagieren können und andererseits aber auch nur so wenig als möglich in das Community-Leben eingreifen.

These 6: Real-Life-Events sind zwar wichtige Elemente zur Belebung der Interaktivität in virtuellen Gemeinschaften, werden aber von Mitgliedern viel unwichtiger eingeschätzt als von Betreibern. Events sollten nicht gehäuft durchgeführt werden. Es ist erfolgversprechender, sich auf wenige, lange vorher angekündigte Events zu beschränken.

These 7: Vor Veränderungen an Darstellungen/Funktionalitäten bzw. vor Erweiterung des Angebots ist es wichtig, den Mitgliedern die Möglichkeit der Mitgestaltung bei dieser Entscheidung zu geben.

These 8: Motivation zur Mitgliedschaft in Communities ist bei Männern eher die Möglichkeit zur unkomplizierten Kontaktaufnahme auf unverbindlicher Basis, ohne den Wunsch diese Kontakte auch in das echte Leben zu überführen. Gründe für die Kontaktaufnahme sind hierbei meist Informationssuchen. Der Aufbau von sozialem Kapital spielt für Männer in Communities eine größere Rolle als für Frauen.

These 9: Motivation zur Mitgliedschaft in Communities ist bei Frauen oftmals schon bestehende Kontakte orts- und zeitungebunden fortzuführen oder neue Kontakte über die Begegnung im Netz hinaus auch im realen Leben zu vertiefen. Das Interesse liegt stärker auf sozialer Interaktion als bei Männern und weniger auf dem Aufbau von sozialem Kapital (bspw. durch Übernahme von Aufgaben in der Community oder häufigem Beitragsschreiben).

These 10: Wichtiger als die permanente Erweiterung des Angebots ist die Einhaltung der Neutralität des Community-Betreibers.

Tabelle 8: 10 Thesen zum Aufbau und Betrieb virtueller Gemeinschaften

Als entscheidende Erkenntnis kann aus den vorliegenden Erörterungen die eindeutige Konzentration sowohl auf Betreiber- als auch auf Mitgliederseite hinsichtlich der gewünschten Performanz, Sicherheit und Aktualität bzw. Hochwertigkeit der Inhalte bezeichnet werden. Die in der Literatur als "typische“ Erfolgsfaktoren für virtuelle Gemeinschaften dargestellten, schneiden in den Untersuchungen zum großen Teil erstaunlich schlecht ab. Nicht die Untergruppen, die Vorzugsbehandlung, die Vergabe von Vergünstigungen oder gar regelmäßige Treffen in der realen Welt stehen im Mittelpunkt des Interesses, sondern die Leistung des Internetaufritts sowohl aus technischer als auch aus inhaltlicher Sicht. Wichtigster Erfolgsfaktor ist bei allen betrachteten Gruppen der sensible
Umgang mit den Daten der Mitglieder, was das Thema Datenschutz (auch bei nicht-kommerziellen Communities) und die sensible Rolle des CommunityManagers und Betreibers betont.

Zusammenfassend läßt sich festhalten: Die Ergebnisse dieser explorativen Untersuchung bedürfen der weitergehenden Fundierung. Trotz der fehlenden Repräsentativität des Samples und trotz der methodischen Restriktionen einer Online-Umfrage bietet es sich an, die erarbeiteten Thesen im Rahmen einer größer angelegten empirischen Untersuchung mit genauerem empirischem Apparat zu überprüfen und hierbei auch noch detaillierter bspw. nach den Arten von Communities oder trennscharf bei den Gruppen der Adressaten zu unterscheiden.

\section{Literatur}

[ArHA95] Armstrong, A.; Hagel III, J. (1995): Real Profits from Virtual Communities. In: The McKinsey Quarterly, (1995) Nr. 3, S. 128-141.

[ArHa96] Armstrong, A.; Hagel III, J. (1996): The Real Value of Online Communities. In: Harvard Business Review, Vol. 74 (1996) Nr. 3, S. 134- 141.

[Bant ${ }^{+}$99] Bantinic, B.; Moser, K.; Puhle, B. (1999): Der WWW-Fragebogengenerator. In: Online Research - Methoden, Anwendungen und Ergebnisse. Hrsg.: W. Bandilla; B. Bantinic; L. Gräf, Verlag für Psychologie Dr. C.J. Hogrefe, Göttingen 1999, S. 93-102.

[Brun $\left.{ }^{+} 00\right]$ Brunold, J.; Merz, H.; Wagner, J. (2000): www.cyber-communities.de - Virtual Communities: Strategie, Umsetzung, Erfolgsfaktoren. mi, Verlag Moderne Industrie, Landsberg/Lech 2000.

[Bull02] Bullinger, H. (2002): Business Communities: professionelles Beziehungsmanagement von Kunden, Mitarbeitern und B2B- Partnern im Internet. 1. Auflage, Galileo Press, Bonn 2002

[Büni $\left.{ }^{+} 81\right]$ Büning, H.; Haedrich, G.; Kleinert, H.; Kuß, A. (1981): Operationale Verfahren der Markt- und Sozialforschung - Datenerhebung und Datenanalyse. Walter de Gruyter GmbH\&Co.KG, Berlin 1981.

[Cass01] Cassiopeia (2001): Handbuch - Cassiopeia Community Application Server, Release 4. o.V., München 2001.

[Döri01] Döring, N. (2001): Virtuelle Gemeinschaften als Lerngemeinschaften? In: http://www.die-frankfurt.de/zeitschrift/32001/positionen4.htm, zugegriffen am 15.09.2002.

[Fahr ${ }^{+}$99] Fahrmeir, L.; Künstler, R.; Pigeot, I.; Tutz, G. (1999): Statistik - Der Weg zur Datenanalyse. 2. Auflage, Springer, Berlin, Heidelberg 1999.

[Figa98] Figallo, C. (1998): Hosting Web Communities: Building Relationships, Increasing Customer Loyalty and Maintaining a Competitive Edge. Wiley Computer Publishing, New York 1998. 
[Gade99] Gadeib, A. (1999): Ansprüche und Entwicklung eines Systems zur Befragung über das World Wide Web. In: Online Research - Methoden, Anwendungen und Ergebnisse. Hrsg.: W. Bandilla; B. Bantinic; L. Gräf, Verlag für Psychologie Dr. C.J. Hogrefe, Göttingen 1999, S. 103-111.

[HaAr97] Hagel III, J.; Armstrong, A. (1997): Net Gain - Expanding markets through virtual communities. Thomas Gabler Verlag, Wiesbaden 1997.

[HaAr99] Hagel III, J.; Armstrong, A. (1999): Net Gain - Profit im Netz. Märkte erobern mit virtuellen Communities. Thomas Gabler Verlag, Wiesbaden 1999.

[Haup99] Hauptmanns, P. (1999): Grenzen und Chancen von quantitativen Befragungen mit Hilfe des Internet. In: Online Research - Methoden, Anwendungen und Ergebnisse. Hrsg.: W. Bandilla; B. Bantinic; L. Gräf, Verlag für Psychologie Dr. C.J. Hogrefe, Göttingen 1999, S. 21-38.

[Kim99] Kim, A.J. (1999): Secret Strategies for Successful Online Communities / Community-Building On The Web. Peachpit Press, Berkeley 1999.

[Mark02] Markus, U. (2002): Integration der virtuellen Community in das CRM: Konzeption, Rahmenmodell, Realisierung. Joseph Eul Verlag, Lohmar; Köln 2002.

[Nies ${ }^{+}$02] Nieschlag, R.; Dichtl, E.; Hörschgen, H. (2002): Marketing. 19., überarbeitete und ergänzte Auflage. Auflage, Duncker\&Humboldt, Berlin 2002.

[Pree00] Preece, J. (2000): Online Communities - Designing Usability, Supporting Sociability. John Wiley and Sons, Chichester, New York, Weinheim, Brisbane, Singapore, Tokio 2000.

[Rhei93] Rheingold, H. (1993): The Virtual Community - Homesteading on the Electronic Frontier. Addison Wesley, Reading, MA 1993.

[ScSc01] Schoberth, T.; "Schrott, G. (2001): Virtual Communities. In: Wirtschaftsinformatik, Vol. 43 (2001) Nr. 5, S. 517-519.

[Schu00] Schubert, P. (2000): Virtuelle Transaktionsgemeinschaften im Electronic Commerce: Management, Marketing und Soziale Umwelt. 2. Auflage, Josef Eul Verlag, Lohmar, Köln 2000.

[Sidi $\left.{ }^{+} 03\right]$ Sidiras, P.; Leimeister, J.M.; Krcmar, H. (2003): Empirische Untersuchung: Erfolgsfaktoren virtueller Gemeinschaften. Arbeitspapier Nr. 114. Universität Hohenheim, Lehrstuhl für Wirtschaftsinformatik, Hohenheim 2003.

[Trom90] Tromsdorf, V. (1990): Erfolgsfaktorenforschung, Produktinnovation und Schnittstelle Marketing-F\&E. Technische Universität Berlin, Berlin 1990.

[Voß00] Voß, W. (2000): Taschenbuch der Statistik. 1. Auflage, Carl Hanser Verlag, München 2000 .

[Zupa99] Zupancic, D. (1999): Ein Blick in die Zukunft virtueller Gemeinschaften. In: IO Management, (1999) Nr. 5, S. S. 42-46. 